\title{
Risk Factors of Fatal Outcome in Patients With COVID-19 Pneumonia
}

\author{
Michaela Cellina, MD (1); Daniele Gibelli, MD, PhD; Carlo Valenti Pittino, MD; \\ Tahereh Toluian, MD; Pietro Marino, MD; Giancarlo Oliva, MD
}

\section{ABSTRACT}

Objectives: The aim of this study was to correlate the clinical, laboratory, and radiographic characteristics of patients with a confirmed diagnosis of coronavirus disease 2019 (COVID-19) disease, with fatal outcome. Methods: We reviewed chest X-ray (CXR) features, clinical, and laboratory data of patients with reverse transcriptase polymerase-chain-reaction confirmed diagnosis of COVID-19 infection. The relationship with mortality was investigated by fitting a logistic regression model.

Results: A total of 246 patients were included (170 males; mean age, $63 \mathrm{y}$ ). Most of the patients had 1 or more comorbidity (62\%); fever (95\%), and cough (60\%) were the most common symptoms; CXR detected abnormalities in $88.6 \%$, mainly showing ground-glass opacities (GGO) (90\%) with bilateral (64\%) and peripheral (46\%) distribution.

Multivariate analysis showed that age ( $P<0.001$; mortality of $59 \%$ in patients $>66$ y old; $5 \%$ at a younger age) and consolidation at CXR ( $P=0.001$; mortality of $11 \%$ with positive CXR; $2 \%$ in those without) represented the 2 most significant independent risk factors of mortality. Chronic pathologies, such as diabetes and chronic obstructive pulmonary disease, and peripheral GGO at CXR also showed a significant correlation with mortality.

Conclusions: We identified predictive factors for the fatal outcome of COVID-19 patients. The prognostic value of these findings can be useful for optimal patient management and resource allocation.

Key Words: coronavirus disease, COVID-19, patient outcome assessment, pneumonia, radiography, viral

S everal cases of pneumonia of unknown etiology have been reported in Wuhan City, Hubei Province, China, in December 2019. ${ }^{1}$ The virus causing the epidemic was identified on January 7 as a new coronavirus (2019-nCoV), and the resulting pneumonia was named by the $\mathrm{WHO}$ as coronavirus disease 2019 (COVID-19). ${ }^{2}$ As of August 31, 2020, a total of $25,118,689$ confirmed cases and of 844,312 deaths have been reported worldwide. ${ }^{3}$

Clinical manifestations of COVID-19 infection are highly variable: serious cases develop severe pneumonia, acute respiratory distress syndrome (ARDS), and multiple organ failures leading to death; nonsevere cases present ordinary symptoms of respiratory system infection; and asymptomatic cases have also been reported. ${ }^{2,4-7}$

One of the issues the involved countries are facing is represented by the very high volume of patients presenting to health centers or hospitals during the outbreak, that overwhelms the health-care resources available, especially the need for critical care support.
Early and effective predictors of clinical outcomes are urgently needed for risk stratification of COVID-19 patients, to help effective patient management and resource allocation. ${ }^{8,9}$

Therefore, our aim was to retrospectively analyze clinical, laboratory, and radiographic characteristics of a consecutive series of patients who presented to our emergency department (ED) with symptoms suspected for COVID-19 infection, with confirmed diagnosis by real-time reverse transcriptase polymerase chain reaction (RT-PCR), to assess the correlation with fatal outcome and to identify variables with prognostic value.

\section{METHODS}

This single-center retrospective study was approved by our institutional review board and performed in a hospital with approximately 60,000 annual ED accesses, located in the center of Milan, in Northern Italy, an area heavily hit by the COVID-19 outbreak. Consent was obtained from the patients involved: after confirmation of COVID-19 positivity, the patients were asked to provide consent regarding the use of 
anonymized demographic and clinical information and submission to the experimental treatments.

All clinical, laboratory, and outcome data were extracted from electronic medical records using a standardized data collection form.

\section{Patients}

We retrospectively analyzed data from our hospital ED electronic database of consecutive patients who presented to our ED with symptoms suspected of COVID-19 infections from 15 February to 30 March 2020.

Inclusion criteria were as follows: (1) patients aged more than $18 \mathrm{y}$, as our hospital was converted to a COVID-19 hub for adults, whereas pediatric patients were treated in other designated institutions; (2) COVID-19 diagnosis confirmation through RT-PCR test performed using nasal and rhinopharyngeal swab specimens; (3) availability of chest X-ray (CXR) and laboratory blood tests executed at patient arrival, and of clinical (symptoms and comorbidities) data assessed at patient admission; (4) patient discharged or deceased.

The clinical outcomes of recovery or death were monitored up to 30 April 3 2020, the final date of follow-up.

\section{Clinical Data}

All data were collected in a standardized Excel electronic database; the variables to insert were decided in consensus by an emergency physician and a radiologist in a preliminary meeting, mainly based only upon our clinical practical experience.

Clinical data and radiological images collection were performed by 2 radiology residents in consensus, blinded to the aim of the study, trained in previous research studies. Data recording was made under the supervision of a radiologist, who checked the correctness of the information.

Patients with missing data were excluded from the analysis. Weekly meetings were held between all study participants to check the progress of data collection.

The following comorbidities were investigated from patients' history: diabetes, arterial hypertension, chronic obstructive pulmonary disease, asthma, cardiovascular disease, cardiac failure, atrial fibrillation, stroke, dementia, obesity, chronic renal insufficiency, malignancy, HIV infection (according to the patients' characteristics collected by the Epidemiology for Public Health-Istituto Superiore di Sanità. ${ }^{10}$ The assumption of angiotensin-converting enzyme inhibitors (ACEi) was recorded, as ACE2 has been shown to be a co-receptor for viral entry for severe acute respiratory syndrome coronavirus 2 (SARS-CoV-2), with increasing evidence of its role in the pathogenesis of COVID-19, and a concern that the use of
ACEI increases expression of ACE2 and patient susceptibility to viral host cell entry and propagation..$^{11,12}$

We collected the following patient symptoms: cough, dyspnea, hemoptysis, chest pain, cutaneous rash, gastrointestinal symptoms, conjunctivitis, fever, and days of fever. Temperature and oxygen saturation at patient arrival were collected, as well as patients' treatments and days of hospitalization.

\section{Blood Tests}

We collected the following blood tests, according to the protocol adopted for suspected COVID-19 patients in our clinical practice, and to the agreement between the emergency physician and the radiologist who decided the variables to collect: leukocytes, neutrophils, lymphocytes, platelets, erythrocytes, D-dimer, aspartate aminotransferase, alanine aminotransferase, lactate dehydrogenase, $\mathrm{C}$-reactive protein, fibrinogen, troponin $\mathrm{T}$, arterial blood gases $(\mathrm{pH}, \mathrm{PaO} 2, \mathrm{PaCO} 2, \mathrm{SaO} 2)$. Creatinine was not included, as we already considered chronic renal insufficiency as a variable.

\section{X-Ray Analysis and Quantification}

All CXRs were acquired in the posteroanterior (PA) + laterolateral projections on a fixed radiographic machine, or in the anteroposterior (AP) projection on a portable radiographic unit, according to our standard acquisition protocols.

Two radiologists (M.C., a radiologist with 9 y of experience; M.O., a radiologist with $7 \mathrm{y}$ of experience) in consensus assessed for each CXR: (1) the presence of lung abnormalities, described as consolidation, ground-glass opacities (GGO), or nodules, according to the Fleischner Society glossary of terms ${ }^{13}$ (Figure 1); (2) their distribution, classified into (i) "peripheral," "central," or "both"; and into (ii) "unilateral" or "bilateral." The presence of pleural effusion was recorded.

A radiographic severity score, according to Wong et al., ${ }^{14}$ was independently assigned by 2 radiologists (M.P., radiologist with $7 \mathrm{y}$ of experience; G.O., radiologist with $25 \mathrm{y}$ of experience), depending on the extent of involvement by consolidation or GGO ( $0=$ no involvement; $1=<25 \% ; 2=25-50 \%$; $3=50-75 \% ; 4=>75 \%$ involvement), for each lung, with a maximum score of 8 for CXR. Some examples are provided in Figure 2. Interobserver agreement was evaluated.

\section{Statistical Analysis}

Values were checked with a 1-sample Kolmogorov-Smirnov test for normality. Interobserver agreement was assessed through Cohen's k correlation coefficient. Kruskal-Wallis $\mathrm{H}$ test and Mann-Whitney U-test were used to evaluate differences between independent groups. Chi-squared test or Fisher's exact test were used to determine significant relationships between categorical variables. Correlations between values were evaluated through Spearman's Correlation 


\section{FIGURE}

Examples of CXR Abnormalities. a: CXR showing a focal GGO involving the middle-upper fields of the right lung (frame). b: CXR showing area of consolidation in the lower right fields (frame). GGO are recognizable in the lower fields of the left lung.

(a)

(b)

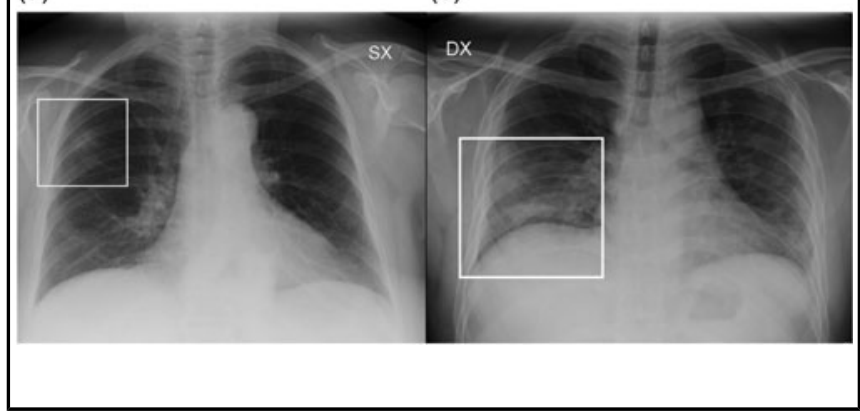

Coefficient: among redundant variables (Spearman coefficient $>$ .80). The relationship between fatal outcome with CXR severity score, radiological features, and clinical and laboratory parameters was investigated fitting a logistic regression model. $P<0.05$ was regarded as statistically significant. To avoid redundancy, we selected between all variables with $P<0.05$ the independent variables (Spearman's $K<0.8$ ) and the most informative among redundant variables, based on receiver operating characteristic analysis. A model was built with the variables thus selected by a backward stepwise model. A chi-squared automatic interaction detection (CHAID) decision tree analysis with CXR score as the user-specified first level was used to detect the fatal outcome. Statistical analysis was performed using SPSS (IBM SPSS Statistics, Version 26.0, IBM Corp).

\section{RESULTS}

\section{Patients}

According to our exclusion criteria, 246 patients were enrolled. Most patients were men (170/246; 69\%), with a mean age of $63 \mathrm{y} ; 154 / 246$ patients $(62 \%)$ have at least 1 chronic disease (Table 1). Upon admission, most patients had fever (234/246; 95\%) and cough (148/246; 60\%); C-reactive protein levels were increased in the $216 / 246(88 \%)$ of patients (Table 2 ).

Most of the patients received a combination of antibiotics and antiviral therapy (152/246 patients; 62\%). Patients' treatments are listed in Table 3.

\section{CXR Analysis and Quantification}

One hundred fifty-six of 246 (63\%) CXR were executed in the posteroanterior projection on a portable X-Ray unit. Two hundred eighteen of 246 (88.6\%) of CXR showed abnormalities.
Most patients (222/246; 90.2\%) showed GGO, with bilateral $(158 / 246,64.2 \%)$ and peripheral $(114 / 246,46.3 \%)$ distribution. The mean radiographic severity score was $3 \pm 2$. Interobserver agreement was excellent (Cohen's K coefficient: 0.901). Overall imaging findings are listed in Table 4.

\section{Fatal Outcome Prediction Univariate Analysis}

The correlation upon univariate analysis of the analyzed variables with fatal outcome is listed in Tables 1, 2, 4.

We observed that different variables significantly correlated with the outcome: sex $(P=0.001)$, and age $(P<0.001)$, various comorbidities, assumption of ACEi $(P<0.001)$, and days of hospitalization $(P<0.001)$.

Analyzing symptoms upon admission, only dyspnea correlated with mortality $(P=0.001)$; fever did not correlate $(P=0.336)$, but the days of fever did $(P<0.001)$.

Among blood tests, C-reactive protein, D-dimer, and fibrinogen were significantly correlated with the outcome $(P=0.001)$.

At CXR, GGO with peripheral distribution, consolidation, pleural effusion, and the severity score showed a significant correlation with fatal outcome $(P<0.001)$.

\section{Multivariate Analysis}

We confirmed the significant correlation with fatal outcome of age $(P<0.001$; odds radio [OR], $1.206 ; 95 \%$ confidence interval $[\mathrm{CI}], 1.12-1.29)$, diabetes $(P<0.001$; OR, 18.890; 95\% CI, 2.9-123.0) and chronic obstructive pulmonary disease ( $P<0.001$; OR, 7.368; 95\%,CI, 1.44-37.60); among radiological features, consolidation $(P=0.004 ; \mathrm{OR}, 5.472 ; 95 \% \mathrm{CI}$, 1.698-17.635) and peripheral GGO $(P=0.14$; OR, 4.208; $95 \%$ CI, 1.343-13.192) were significantly correlated with fatal outcome at multivariate analysis. See Table 5 for results.

Lymphocytes ( $P=0.726$; OR, 0.634; 95\% CI, 0.050-8.109), troponin $\mathrm{T}(P=0.164 ; \mathrm{OR}, 1.698 ; 95 \% \mathrm{CI}, 0.805-3.581)$, C-reactive protein $(P=0.901 ; \mathrm{OR}, 1.120 ; 95 \% \mathrm{CI}, 0.187$ 6.722), D-dimer $(P=0.292$; OR, 2.883; 95\% CI, 0.403 20.632), and fibrinogen $(P=0.476 ; \mathrm{OR}, 1.946 ; 95 \% \mathrm{CI}$, $0.312-12.118)$ did not show statistically significant correlation with the outcome at multivariate analysis.

Multivariate analysis using the CHAID method showed that the 2 most significant variables in predicting a fatal outcome were the age of the patient and the presence of consolidation. Mortality was $59 \%$ in patients $>66$ y old, and $5 \%$ in patients younger than that; in the latter group, mortality was $11 \%$ in patients presenting with consolidation, and $2 \%$ in those without it. 


\section{TABLE 1}

\section{Role of the PCSS in Identifying Issues and Solutions}

\section{Issue}

Concern about discharging patients with high oxygen requirements with exertion

Patients unable to discharge to care facilities due to COVID-19

Lack of standardization in discharge care for patients with COVID-19

Respiratory therapy overwhelmed by need for home oxygen to be completed coupled with increase in tracheostomy care and complex respiratory issues
Group Affected

Patients with oxygen levels $>2 \mathrm{~L} / \mathrm{min}$ with exertion

Patients no longer meeting inpatient criteria but unable to return home

All COVID-19 positive inpatients

Respiratory therapy

\section{PCSS Action}

Group coordination to develop a protocol involving multiple groups

Group coordination to determine what care facilities would deem acceptable acceptance criteria

Group coordination between extensive stakeholders including outpatient providers, telehealth monitoring, and respiratory therapy

Group creation of a new group of backup respiratory therapists
Result

New Home Oxygen Pathway with specific attention to this group of patients

Negative COVID-19 nasopharyngeal swabs X2 was considered acceptable risk and patients then were transferred successfully to care facilities

Standardized discharge pathway easily orderable through the EMR with the additional benefit of standardized "dot phrases" allowing standardized verbiage in the chart and for home-going

Allows main respiratory therapists to provide direct patient care while back-up therapists completed the challenging home oxygen process

Examples of CXR Severity Score Assignment. a: CXR showing focal bilateral GGO in the lower fields (frames). On both left and right lung, the involvement was $<25 \%$; therefore, the CXR severity score assigned was 1 for each lung, with a global score of 2. b: CXR showing bilateral parenchymal opacities (frames): a huge area of consolidation in the middle-lower left fields with contextual air bronchogram, while a focal area of GGO is recognizable in the upper fields of the left lung; the extension on the left side was $>\mathbf{5 0} \%$ (score 3 ), whereas the involvement on the right side was $<\mathbf{2 5 \%}$ (score 1 ); therefore, the overall score was $3+1=4$. c: CXR showing bilateral involvement, with mixed areas of GGO and consolidation (frames) involving all the lung fields. On both left and right lung, the involvement was $>75 \%$ (score 4); therefore, the global score was $4+4=8$.

(a)

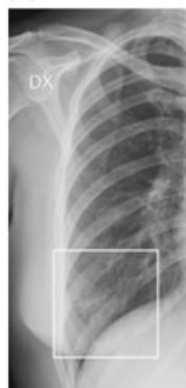

(b)

(c)

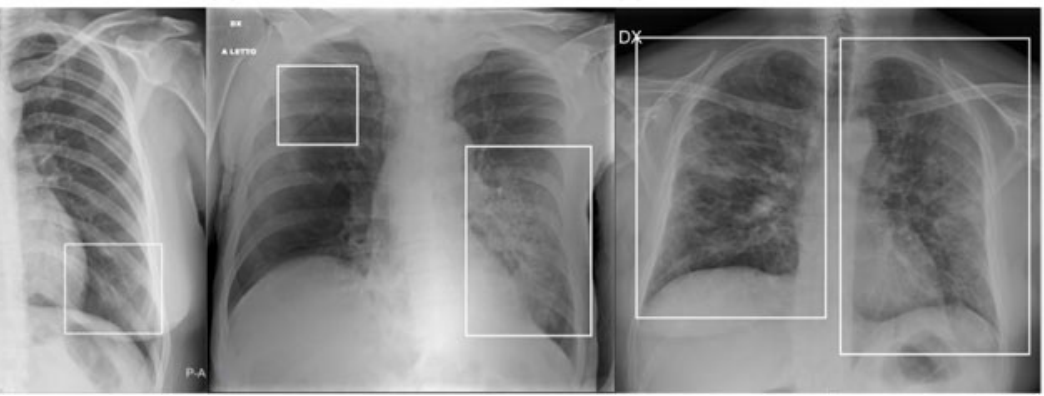

\section{DISCUSSION}

In our retrospective study, we observed that the fatal outcome had a significant correlation with sex, age, various comorbidities, the assumption of ACEi, and days of hospitalization $(<0.001)$. The only symptom upon admission with a significant correlation was dyspnea, whereas, among blood tests, mainly troponin T, C-reactive protein, D-dimer, and fibrinogen significantly correlated with the outcome.
Regarding radiological variables, consolidation, peripheral GGO, pleural effusion, and the severity score showed a significant correlation.

At the logistic regression model, only age, diabetes, chronic obstructive pulmonary disease, peripheral GGO, and consolidation showed significance as independent risk factors for fatal outcome in COVID-19 infection. 
Patients Signs, Symptoms, and Laboratory Tests and Their Correlation With Fatal Outcome

\section{Sign and Symptoms}

Cough

Dyspnea

Hemoptysis

Chest pain

Cutaneous rash

Gastrointestinal symptoms

Conjunctivitis

Fever

Days of fever

Temperature

Oxygen saturation

Blood tests

Leukocytes

Neutrophils

Monomorphous \%

Polymorphus \%

Lymphocytes

Platelets

Erythrocytes

D-dimer

Aspartate aminotransferase

Alanine aminotransferase

Lactate dehydrogenase

C-reactive protein

Fibrinogen

Troponin T

Arterial blood gas

\section{Patients Number/total (\%)}

$148 / 246(60.1 \%)$

$120 / 246(48.8 \%)$

$2 / 246(0.8 \%)$

$12 / 246(4.9 \%)$

$2 / 246(0.8 \%)$

$52 / 246(21.14 \%)$

$4 / 246(1.63 \%)$

$234 / 246(95.1 \%)$

$6.7 \pm 6(3.5 \%)$

$37.9 \pm 0.8^{\circ} \mathrm{C}$

$91.5 \pm 7.7$

7.3 3.9 109/L (NR 4.19-9.35 109/L)

$5.4 \pm 3.9109 / \mathrm{L}$ (NR 1.91-6.23 10\%/L)

$24.87 \pm 13 \%$

$75.15 \pm 12.9 \%$

$1.4 \pm 1.4 \times 10^{9} / \mathrm{L}\left(\mathrm{NR} 1.13-3.37 \times 10^{9} / \mathrm{L}\right)$

$227 \pm 111 \times 10^{9} / \mathrm{L}$ (NR. 169-359 109/L)

$4.7 \pm 0.8 \times 10^{12} / \mathrm{L}\left(\mathrm{NR} 4.13-5.15^{*} 10^{\wedge} 12 / \mathrm{L}\right)$

$1532 \pm 5600 \mathrm{ng} / \mathrm{mL}(\mathrm{NR} 250-500 \mathrm{ng} / \mathrm{mL})$

$54.1 \pm 38.2 \mathrm{U} / \mathrm{L}$ (NR 10-35 U/L)

$44.6 \pm 41 \mathrm{U} / \mathrm{L}$ (NR. 10-33 U/L)

$353.6 \pm 148.9 \mathrm{U} / \mathrm{L}$ (NR 135-225 U/L)

$99.8 \pm 94.3 \mathrm{mg} / \mathrm{L}(\mathrm{NR} 0-5 \mathrm{mg} / \mathrm{L})$

$633.86 \pm 88.82$ (V.N. $270-470 \mathrm{mg} / \mathrm{dL}$ )

$32.03 \pm 38.29$ (V.N. 0-14 ng/L)

pH 7.46 $\pm 0.056 \mathrm{PaO} 263.68 \pm 18.85 \mathrm{PaCO} 233.40 \pm 8.94 \mathrm{SaO} 291.16$ $\pm 11.29$

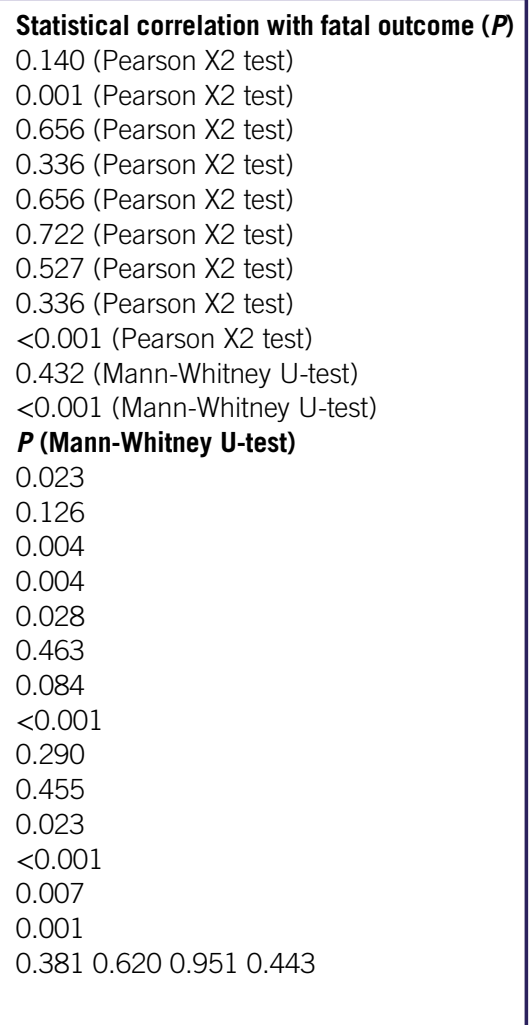

Statistical correlation with fatal outcome $(P)$

0.140 (Pearson X2 test)

001 (Pearson X2 test)

0.656 (Pearson $X 2$ test)

0.722 (Pearson $\mathrm{X} 2$ test)

0.527 (Pearson $\times 2$ test)

$<0.001$ (Mann-Whitney U-test)

$\boldsymbol{P}$ (Mann-Whitney U-test)

0.004

0.028

0.290

0.455

$<0.001$

0.007

0.3810 .6200 .9510 .443

Abbreviation: NR, normal range.

\section{TABLE 3}

\section{Patients Treatment During Hospitalization}

Non-invasive ventilation

Invasive ventilation

Hydroxychloroquine

Antiviral treatment (lopinavir/ritonavir)

Antibiotic treatment (Azithromycin)

Tocilizumab

Paracetamol

Dexamethasone

Methylprednisolone

Patients

Number/total (\%)

$86 / 246(34.9 \%)$

$26 / 246(10.5 \%)$

$206 / 246(83.7 \%)$

$166 / 246(67.5 \%)$

$218 / 246(88.6 \%)$

$46 / 246(18.7 \%)$

$148 / 246(60.1 \%)$

$88(35.7 \%)$

$16(6.5 \%)$

Understanding of the new coronavirus SARS-CoV-2 and COVID-19 is still evolving.

Disease severity varies broadly, with an estimated $81 \%$ of confirmed cases showing only mild disease, $14 \%$ severe pneumonia (defined as dyspnea, hypoxia, or $>50 \%$ lung involvement on imaging within 24-48 h), and 5\% critical disease (defined as respiratory failure, shock, or multiorgan failure).,15 The case-fatality rate ranged from 0.7 to $14 \%$, varying by location,

the intensity of transmission, and the time of infection. The risk factors of mortality are still unclear: most of the fatal cases were observed in older patients or patients with underlying medical comorbidities, ${ }^{15-20}$ but other clinical factors still need to be identified.

In line with our results, a study on 150 patients in Wuhan, China, 31 demonstrated a significant difference in age between the dead and discharged patients $(P<0.001)$, but no difference in the sex ratio. Moreover, $63 \%$ of patients in the death group and $41 \%$ of patients in the discharge group had underlying diseases, with statistically significant differences; especially patients with cardiovascular diseases showed a significantly increased risk of death $(P<0.001)$. Significant differences between the 2 groups were also observed in white blood cell counts, absolute values of lymphocytes, platelets, albumin, total bilirubin, blood urea nitrogen, blood creatinine, myoglobin, cardiac troponin, C-reactive protein, and interleukin-6; however, the regression analysis was not performed to assess their role as independent risk factors.

Low white cells and platelet count, high D-dimer values, and high pro-thrombin time showed a correlation with mortality. ${ }^{8,20}$ Significantly higher levels of aspartate 


\begin{tabular}{|c|c|c|}
\hline \multicolumn{3}{|c|}{$\begin{array}{l}\text { Radiographic Findings and Their Distribution, and } \\
\text { Radiographic Severity Score in Our Patient Population } \\
\text { and Correlation With the Fatal Outcome }\end{array}$} \\
\hline & $\begin{array}{l}\text { Patients } \\
\text { number/total (\%) }\end{array}$ & $\begin{array}{l}\text { Statistical correlation with } \\
\text { fatal outcome }(P)\end{array}$ \\
\hline $\begin{array}{l}\text { Presence of X-ray } \\
\text { abnormalities }\end{array}$ & $\begin{array}{l}218 / 246 \\
(88.6 \%)\end{array}$ & $<0.001$ (Pearson X2 test) \\
\hline $\begin{array}{l}\text { Radiographic } \\
\text { findings }\end{array}$ & & Pearson $\mathrm{X} 2$ test \\
\hline GGO & $\begin{array}{l}222 / 246 \\
(90.2 \%)\end{array}$ & $<0.001$ \\
\hline Consolidation & $106 / 246(43 \%)$ & $<0.001$ \\
\hline Nodules & $0 / 246(0 \%)$ & \\
\hline Pleural effusion & $26 / 246(10.5 \%)$ & $<0.001$ \\
\hline Distribution & & $<0.001$ (Pearson X2 test) \\
\hline Peripheral & $\begin{array}{l}114 / 246 \\
(46.3 \%)\end{array}$ & \\
\hline Central & $30 / 246(12.2 \%)$ & \\
\hline Peripheral+central & $80 / 246(32.5 \%)$ & \\
\hline Side & & $<.001$ (Pearson X2 test) \\
\hline Unilateral & $66 / 246(26.8 \%)$ & \\
\hline Bilateral & $\begin{array}{l}158 / 246 \\
(64.2 \%)\end{array}$ & \\
\hline Severity Score & $3.28 \pm 2.02$ & $<.001$ (Pearson X2 test) \\
\hline
\end{tabular}

Abbreviation: GGO, ground glass opacity.

aminotransferase, urea, creatinine, creatinine kinase, and lactate dehydrogenase were found in dead patients than in survivors. $8,21,22$

In our study, no blood tests showed significance as an independent risk factor of mortality.

Other authors considered different variables as predictors of outcome in COVID-19 patients.

Chen et al. ${ }^{4}$ applied a severity score for other types of viral pneumonia, called "MuLBSTA score" 23 to predict the risk of mortality in COVID-19 patients: this score system includes multilobar infiltration at imaging, lymphopenia, bacterial co-infection, smoking history (acute or previous), hypertension, and age ( $\geq 60 \mathrm{y}$ ). The authors observed that, in their population of 99 cases in Wuhan, China, the characteristics of patients who died were in line with the MuLBSTA score, but claimed the need of further studies to assess the applicability of this score in the prediction of the risk of mortality in COVID-19 patients. The evidence of age as a risk factor for unfavorable outcome is in line with our results.

In a retrospective cohort of 1590 hospitalized patients with COVID-19 throughout China, ${ }^{24}$ age $>65 \mathrm{y}$, and a history of coronary heart disease and cerebrovascular disease were significantly associated with nonsurvival of patients; the presence of dyspnea, high procalcitonin $(>0.5 \mathrm{ng} / \mathrm{mL})$, and aspartate aminotransferase level ( $>40 \mathrm{U} / \mathrm{L}$ ) proved to be independent risk factors of fatal outcome.

From a radiological point of view, the main radiological characteristics of COVID-19 pneumonia are the alveolar disease represented by GGO (40-91\%), with bilateral distribution $(28-91 \%)$, and a prevalent involvement of peripheral areas $(22-71 \%)$. This triad seems to be more common in the middle stages of the disease. ${ }^{25,26}$ These findings were confirmed to be the most common presentation in our study and a peripheral presentation was also found to be correlated to a fatal outcome at univariate analysis; this result, confirmed at multivariate analysis, suggests an underlying mechanism different from simple alveolar damage may link GGO and mortality, as shown in a work by Lang et al. ${ }^{27}$

The evidence at univariate analysis of a significant correlation of pleural effusion with the final outcome is in line with the results previously reported by Li et al, who suggested the role of this imaging finding as an index of severe disease. $^{28}$

Pan et $\mathrm{al} .{ }^{25}$ and Shi et al. ${ }^{26}$ reported that consolidation seems to be more common in the later stages; the correlation found in our work at univariate analysis between consolidation and fatal outcome can be thought to be due to patients presenting in a later stage of the disease having a worse outcome than patients presenting sooner. Nevertheless, multivariate analysis showed consolidation to be an independent predictor of mortality.

In our study, a significant correlation was found between a fatal outcome and the CXR severity score: a similar result was obtained in a retrospective study on 100 hospitalized patients with COVID-19 infection by Borghesi and Maroldi, in which the quantitative analysis of CXR, based on the type of lung abnormalities, significantly correlated with the final outcome, with severity score higher $(P \leq 0.002)$ in patients who died than those who were discharged. ${ }^{29}$

The value of the CXR severity score upon admission had also been proved in middle-aged patients, demonstrating that a severity score CXR score $\geq 3$ was an independent predictor for intubation, and was higher in patients who died than in survivors, even if without a statistically significant difference. ${ }^{30}$

In our case series, most of the patients were treated with hydroxychloroquine, an analog of chloroquine with a better safety profile, and fewer drug interactions, that showed in vitro antiviral activity against SARS-CoV-2. ${ }^{31}$ As the epicenter of COVID-19 shifted from China to Europe, the use of hydroxychloroquine was recommended also by European authors as a possible prophylaxis and curative treatment for COVID-1932,33; therefore, we adopted its use in our COVID-19 treatments. The effective benefits 
Results of the Multivariate Analysis

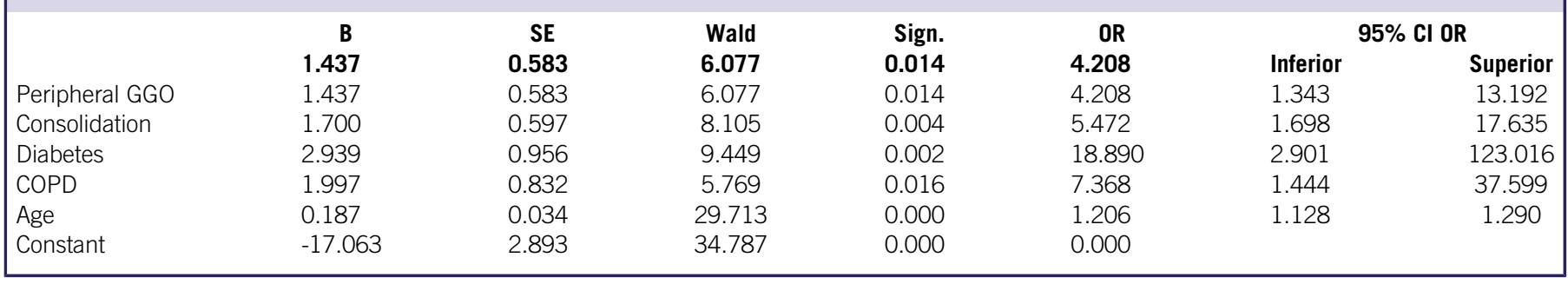

Abbreviations: CI, confidence interval; COPD, chronic obstructive pulmonary disease; GGO, ground glass opacities; OR, odds ratio; SE, standard error; Sign., significance.

form this treatment still remain unclear, ${ }^{34}$ and its use is now limited to clinical studies. ${ }^{35}$

This study has different limitations: first, related to the retrospective nature of this study. This is a single-center study with a relatively limited number of patients. We excluded many patients because, even if the reported symptoms were suspected for COVID-19 infection, the diagnosis was not confirmed by the RT-PCR; moreover, we excluded patients with data missing due to the retrospective nature of the study, transfer to and from other hospitals, and length of hospitalization. Even if many variables were included, according to our routine clinical practice, other variables could be assessed (ie, smoking history, other laboratory tests, and such); moreover, other possible clinical outcomes, such as the need for mechanical ventilation, can be considered.

Another limitation was that the therapy was inhomogeneous due to the absence of specific treatment guidelines at the time of the study; however, the clinical benefits of most treatments tested in COVID-19 patients remain controversial. Due to the complexity of the current clinical situation, studies including large case series and different variables are desirable and needed.

\section{CONCLUSIONS}

Predictors of a fatal outcome in COVID-19 cases included age, the presence of underlying diseases (diabetes and chronic obstructive pulmonary disease), and the radiological evidence of peripheral GGO and consolidation.

We identified some variables associated with unfavorable outcome in patients affected by COVID-19 pneumonia. Identifying the presence of risk variables upon patient admission can improve patient management and the appropriate allocation of available health-care resources.

\section{About the Authors}

Department of Radiology, ASST Fatebenefratelli Sacco, Milano, Italy (Dr Cellina, Dr Oliva); Dipartimento di Scienze Biomediche per la Salute, Università degli Studi di Milano, Milano, Italy (Dr Gibelli); Scuola di Specializzazione in Radiodiagnostica, Università degli Studi di Milano, Milano, Italy (Dr Valenti Pittino, Dr Toluian) and Department of Emergency Medicine, ASST Fatebenefratelli Sacco, Milano, Italy (Dr Marino).

Correspondence and reprint requests to Michaela Cellina, ASST Fatebenefratelli Sacco, Milano Piazza Principessa Clotilde 3, 20121, Milano, Italy (e-mail: michaela.cellina@asst-fbf-sacco.it).

\section{Conflicts of Interest}

Authors declare no conflict of interest.

\section{REFERENCES}

1. Lu H, Stratton CW, Tang YW. Outbreak of pneumonia of unknown etiology in Wuhan, China: the mystery and the miracle. J Med Virol. 2020;92(4):401-402.

2. Huang C, Wang Y, Li X, et al. Clinical features of patients infected with 2019 novel coronavirus in Wuhan, China. Lancet. 2020. doi: 10.1016/ S0140-6736(20)30183-5

3. WHO. WHO coronavirus disease (COVID-19) dashboard. https:// covid19.who.int/. Accessed October 8, 2020.

4. Chen N, Zhou M, Dong X, et al. Epidemiological and clinical characteristics of 99 cases of 2019 novel coronavirus pneumonia in Wuhan, China: a descriptive study. Lancet. 2020;395:507-513. doi: 10.1016/ S0140-6736(20)30211-7

5. Wang D, Hu B, Hu C, et al. Clinical characteristics of 138 hospitalized patients with 2019 novel coronavirus-infected pneumonia in Wuhan, China. JAMA. 2020;323(11):1061-1069. doi: 10.1001/jama.2020.1585

6. Hu Z, Song C, Xu C, et al. Clinical characteristics of 24 asymptomatic infections with COVID-19 screened among close contacts in Nanjing, China. Sci China Life Sci. 2020;63(5):706-711. doi: 10.1007/s11427020-1661-4

7. Nishiura H, Kobayashi T, Miyama T, et al. Estimation of the asymptomatic ratio of novel coronavirus infections (COVID-19). Int J Infect Dis. 2020;94:154-155. doi: 10.1016/j.ijid.2020.03.020

8. Zhang L, Yan X, Fan Q, et al. D-dimer levels on admission to predict inhospital mortality in patients with Covid-19. J Thromb Haemost. 2020;10. 1111/jth.14859. doi: 10.1111/jth.14859

9. Guan WJ, Ni ZY, Hu Y, et al. Clinical characteristics of coronavirus disease 2019 in China. N Engl J Med. 2020;382(18):1708-1720. doi: 10.1056/ NEJMoa2002032

10. Epidemiology for Public Health. Istituto Superiore di Sanità. Characteristics of COVID-19 patients dying in Italy. https://www.epicentro.iss.it/coronavirus/ sars-cov-2-decessi-italia. Accessed July 22, 2020.

11. Zhou P, Yang X-L, Wang X-G, et al. A pneumonia outbreak associated with a new coronavirus of probable bat origin. Nature. 2020; 579(7798):270-273. doi: 10.1038/s41586-020-2012-7 
12. Patel AB, Verma A. COVID-19 and angiotensin-converting enzyme inhibitors and angiotensin receptor blockers: what is the evidence? JAMA. 2020;323(18):1769-1770. doi: 10.1001/jama.2020.4812

13. Hansell DM, Bankier AA, MacMahon H, et al. Fleischner Society: glossary of terms for thoracic imaging. Radiology. 2008;246(3):697-722. doi: 10.1148/radiol.2462070712

14. Wong HYF, Lam HYS, Fong AH-T, et al. Frequency and distribution of chest radiographic findings in COVID-19 positive patients. Radiology. 2019;296(2):E72-E78. doi: 10.1148/radiol.2020201160

15. Wu Z, McGoogan JM. Characteristics of and Important lessons from the coronavirus disease 2019 (COVID-19) outbreak in China. JAMA. 2020;323(13):1239-1242. doi: 10.1001/jama.2020.2648

16. Lai CC, Shih TP, Ko WC, et al. Severe acute respiratory syndrome Coronavirus 2 (SARS-CoV-2) and coronavirus disease-2019 (COVID19): the epidemic and the challenges. Int J Antimicrob Agents. 2020;55(3):105924. doi: 10.1016/j.ijantimicag.2020.105924

17. Zhou F, Yu T, Du R, et al. Clinical course and risk factors for mortality of adult inpatients with COVID-19 in Wuhan, China: a retrospective cohort study. Lancet. 2020;395(10229):1054-1062. doi: 10.1016/S01406736(20)30566-3

18. Li X, Xu S, Yu M, et al. Risk factors for severity and mortality in adult COVID-19 inpatients in Wuhan. J Allergy Clin Immunol. 2020;146(1): 110-118. doi: 10.1016/j.jaci.2020.04.006

19. Du RH, Liang LR, Yang CQ, et al. Predictors of mortality for patients with COVID-19 pneumonia caused by SARS-CoV-2: a prospective cohort study. Eur Respir J. 2020;55(5):2000524. doi: 10.1183/13993003.00524-2020

20. Wang L, He W, Yu X, et al. Coronavirus disease 2019 in elderly patients: characteristics and prognostic factors based on 4-week follow-up. J Infect. 2020;80(6):639-645. doi: 10.1016/j.jinf.2020.03.019

21. Tan L, Wang Q, Zhang D, et al. Lymphopenia predicts disease severity of COVID-19: a descriptive and predictive study. Signal Transduct Target Ther. 2020;5:33. doi: 10.1038/s41392-020-0148-4.

22. Yang Z, Shi J, He Z, et al. Predictors for imaging progression on chest CT from coronavirus disease 2019 (COVID-19) patients. Aging (Albany NY). 2020;12(7):6037-6048. doi: 10.18632/aging.102999

23. Guo L, Wei D, Zhang X, et al. Clinical features predicting mortality risk in patients with viral pneumonia: the MuLBSTA score. Front Microbiol. 2019;10:2752. doi: $10.3389 /$ fmicb.2019.02752
24. Chen R, Liang W, Jiang M, et al. Risk factors of fatal outcome in hospitalized subjects with coronavirus disease 2019 from a nationwide analysis in China. Chest J. 2020;158(1):97-105. doi: 10.1016/j.chest. 2020.04.010

25. Pan F, Ye T, Sun P, et al. Time course of lung changes on chest $C T$ during recovery from 2019 novel coronavirus (COVID-19) pneumonia. Radiology. 2020;200370. doi: 10.1148/radiol.2020200370

26. Shi $\mathrm{H}$, Han X, Jiang N, et al. Radiological findings from 81 patients with COVID-19 pneumonia in Wuhan, China: a descriptive study. Lancet Infect Dis. 2020;20:425-434. doi: 10.1016/s1473-3099(20)30086-4

27. Lang M, Som A, Mendoza DP, et al. Hypoxaemia related to COVID-19: vascular and perfusion abnormalities on dual-energy CT. Lancet Infect Dis. 2020;S1473-3099(20)30367-4. doi: 10.1016/S1473-3099(20)30367-4

28. Li K, Wu J, Wu F, et al. The clinical and chest CT features associated with severe and critical COVID-19 pneumonia. Invest Radiol. 2020;55(6):327331. doi: 10.1097/RLI.0000000000000672

29. Borghesi A, Maroldi R. COVID-19 outbreak in Italy: experimental chest $\mathrm{X}$-ray scoring system for quantifying and monitoring disease progression. Radiol Med. 2020;125(5):509-513. doi: 10.1007/s11547-020-01200-3

30. Toussie D, Voutsinas N, Finkelstein M, et al. Clinical and chest radiography features determine patient outcomes in young and middle age adults with COVID-19. Radiology. 2020;297(1):E197-E206. doi: 10.1148/radiol. 2020201754

31. Liu J, Cao R, Xu M, et al. Hydroxychloroquine, a less toxic derivative of chloroquine, is effective in inhibiting SARS-CoV-2 infection in vitro. Cell Discov. 2020;6:16. doi: 10.1038/s41421-020-0156-0

32. Colson P, Rolain JM, Raoult D. Chloroquine for the 2019 novel coronavirus SARS-CoV-2. Int J Antimicrob Agents. 2020;55(3):105923. doi: $10.1016 /$ j.ijantimicag.2020.105923

33. Colson P, Rolain JM, Lagier JC, et al. Chloroquine and hydroxychloroquine as available weapons to fight COVID-19. Int J Antimicrob Agents. 2020;55(4):105932. doi: 10.1016/j.ijantimicag.2020.105932

34. Funck-Brentano C, Salem JE. Chloroquine or hydroxychloroquine for COVID-19: why might they be hazardous? Lancet. 2020;S01406736(20)31174-0. doi: 10.1016/S0140-6736(20)31174-0

35. Agenzia Italiana del Farmaco. https://www.aifa.gov.it/web/guest/-/aifasospende-l-autorizzazione-all-utilizzo-di-idrossiclorochina-per-il-trattamentodel-covid-19-al-di-fuori-degli-studi-clinici. Accessed October 8, 2020. 